\section{IMPETIGO CONTAGIOSA AS SEEN IN SCHOOLS.}

By A. I. SIMEY, M.D. CAMB., M.R.C.P.LOND., MEDTCAL OFFICER TO RUGRY SCHOOL AND SENIOR PHYSICLAN TO THE HOSPITAL OF ST. CROSS, RUGBY.

Impetigo contagiosa, commonly known in boarding schools as "Scrum Pox," is a very highly contagious skin disease, affecting chiefly the face and scalp, but at times spreading to other parts of the body and to the limbs. Bacteriological investigations seem to point to the primary infection being streptococcal, but by the time treatment is sought staphylococci are often predominant, chiefly $S$. aureus. In many cases the organism is introduced into an abrasion or laceration, but sometimes this is not evident, and careful observation reveals a small pimple capped by a still more tiny vesicle, which enlarges rapidly and eventually gives rise to the characteristic honey-coloured lesion. In some cases the disease is inoculated into acne spots from which the top has been scratched, and not infrequently impetiginous and pustular spots coexist side by side. One fact is certain-viz., that infection is introduced from without, and a common source which does not receive due recognition is the hair and scurf.

Infection during Football.

When the scalp is scurfy and the hair allowed to grow too long, and especially if it is treated with pomades, it acts as a germ-trap, and, when rubbed against an infected face or jersey in a football scrummage, it likewise becomes infected. In such cases, if the face-especially the folds between the ears and the temples-are not washed after the hair is brushed, a layer of infected scurf is deposited, and a slight tear of the ear, such as is very common in football, gives the germ an easy port of entry into the superficial layers of the skin. 'I'his causes irritation, and leads to scratching, and an ample supply of streptococci finds its way under the finger-nails, sometimes causing a whitlow, and frequently giving rise to further spots by direct inoculation of the skin of the face. Shaving, especially with a blunt tearing razor, often prepares the way for inoculation from the spots, scalps, or jerseys of other players. It would be well to abstain from shaving within 12 hours of a football match.

In days when less attention was paid to hygiene, the disease was, no doubt, spread by the use of common towels and sponges. It is not at all uncommon to find a localised outbreak following a football match, due to the presence of one boy suffering from a single unrecognised spot; hence it is most important that, in a school, every spot which looks in the least suspicious should be shown to the medical officer so that he may segregate the infected person. It is also most important that, during football terms especially, schoolboys should be made to wear their hair short and have their scalps regularly washed or shampooed; they should be taught to begin the morning toilet by brushing the hair thoroughly, and after that never to omit vigorous washing of the face, especially around the ears, with soap and hot water. Special attention should also be paid to the finger-nails, which should be kept cut short and scrupulously clean.

Predisposition and Treatment.

Certain individuals and certain ages are, no doubt, especially prone to impetioinous infections. Some persons suppurate with the least provocation, especially during the damp, sunless months of the year when the skin does not act so freely as it does in summer, and impetigo is very common amongst adolescents. In spite of this, the disease is preventable to a large extent; regular hot baths with a liberal use of soap and vigorous rubbing with a rough towel is a necessary precaution. There is no disease so capricious in its response to treatment; remedies which suit one patient fail with another. Removal of the crusts and application of dilute nitrate of mercury ointment, if carried out regularly and antiseptically, is usually all that is necessary, but in some cases these remedies fail, and no sooner is one spot cured than another appears. Early application of iodine ( 2 per cent. in S.V.R.) often aborts the development of a spot. Some lesions are best kept undisturbed and dry throughout, whilst in many cases a most efficacious remedy is the application of weak lysol or boric fomentations, keeping the part well covered.

Constitutional measures must not be neglected including regular exercise; the diet should include a liberal supply of fresh milk, butter, green vegetables, and ripe uncooked fruit. Of medicines, cod-liver oil and iron, extract of malt, and aperients containing sulphur are the most efficacious, whilst a short stay at a bracing seaside resort will often turn the scale in favour of recovery, even in most obstinate cases, Vaccine treatment is sometimes most efficacious, especially a mixed vaccine containing streptococcus and mixed staphylococcus strains. Injections into the muscles of colloidal manganese have been used by some with good effect.

In conclusion. I would advocate that the disease be tackled in schools by a careful common-sense but strict attention to toilet, especially of the hair and scalp and fingers, and by immediate and cornpulsory notification of every suspicious spot to the medical officer.

\section{OBSERVATIONS ON AN EXTENSIVE HUMAN} INFECTION BY

\section{SARCOPTIC MANGE OF THE HORSE.}

\section{BY R. A. S. MACDONALD, B.Sc., M.R.C.V.S.}

THE circumstances of this outbreak are peculiar, though not entirely unprecedented. A class of veterinary students was engaged upon the performance of post-mortem operations on a horse. The horse had a history of generalised sarcoptic mange, the disease having gained such a hold that it was considered an unsound economic proposition to proceed further with treatment. It was destroyed one Saturday morning at $10 \mathrm{~A} . \mathrm{M}$. and thereafter was left lying upon a concrete floor while the students in question palpated, dissected, and operated upon the carcass for a period of time which varied with the individual from two to five hours.

Contact.

Of the students who had any contact with the carcass in question, approximately 53 per cent. became infected. On the other hand, the non-infected individuals were either those who were engaged upon the head and neck (which parts had been cured by the previous treatment) or those who had been little more than onlookers, in that they had been engaged in swabbing incisions, \&c., to assist the actual operators. In short, of these students whose hands and clothing came into direct and prolonged contact with the infected carcass 100 per cent. complained of subsequent symptoms.

\section{Symptoms.}

The most constant symptoms common to every case were :-

(a) Itch of a most pronounced and continuous nature, accentuated by surface warmth, induced by exercise, by lying in bed, and by friction in the process of inunction of the affected areas.

(b) A papular eruption. This was present in all the affected cases at the site of contact with the carcassi.e., forearms, ankles, and calves of the legs. The hands in most cases, though not in all, escaped infection, no doubt owing to washing with carbolic soap immediately after, or in some cases during, the work on the dead subject. In some cases the forearms escaped while the ankles were affected or vice versa. Lesions on the shoulders, upper arms, trunk, and thighs occurring in some of the students were in all cases secondary, there being no direct track of infection on the skin The papules were the size of a large pin's head, of a fiery-red colour, and were very slightly elevated above 
the surface of the surrounding skin. They seemed to correspond to hair-follicles, and the crops were luxurious. It is perhaps significant that in Case A (referred to later) the interdigital spaces were the seat of the infection; here also the papules were more circumscribed, more elevated, and slightly pointed.

Treatment.

From the strictly scientific point of view it may be regarded as somewhat unfortunate that the affected individuals were all well versed in the life-history and pathogenicity of Sarcoptes scabiei, and that the morbid conditions were therefore rapidly recognised, and curative measures taken accordingly. It might be noted here that the sooner the infection was realised the more easily was a cure effected. Thus, in B's case, after two hours' work on the carcass he became aware of an intense itching on the right forearm, with some slight hyperæmia of the part. He at once painted the area with weak tincture of iodine and had no further trouble at that point. Eight hours later similar lesions appeared upon the calves of the legs. Tinct. iodin. was again applied and the eruption disappeared in one day. Case $\mathrm{C}$ became aware of infection before leaving the establishment at 1 P.M., but considered the violent itch to be a form of scabiophobia. At 2.30 P.M. he examined the lateral aspect of the calf (an area of contact with the carcass) and found the cause of the itch to be the typical papular eruption. He at once took a hot bath and scrubbed the affected area for some minutes with a stiff nail-brush. The area was dressed with 1 in 10 sulphur ointment until two days after infection, by which time the irritation had disappeared, although the eruption did not entirely subside until two days later. Case A itched during the first day and only awoke to the situation on the second day. He became ultimately affected with the typical papular eruption in the interdigital spaces, all over the arms, as well as almost all over the legs. This is one of the few cases with sequelæ.

The treatment adopted varied in detail but, generally speaking, consisted in scrubbing the whole body and especially the affected areas in a hot bath to which was added some coal-tar disinfectant or washing-soda. This was followed by inunction with a preparation in which sulphur was the chief ingredient, adjuvants being salicylic acid, ammonium sulphide, ol. picis, \&c. The presumably infected clothing was in most cases cast off at once pending disinfection. In connexion with the latter point one notes that Cases $D$ and $E$ report no trouble on the Saturday of infection nor on the following Sunday-when different suits were worn - but on the Monday, when the suits worn on the Saturday were resumed, they became typically infected. Case $\mathrm{F}$ applied pure creosote to each papule with the aid of a match-stick. This treatment relieved the itch but had no effect on the eruption, and on the Wednesday he fell into line with the hot scrubbing and sulphur applications.

\section{Sequelce.}

A few cases reported a slight dermatitis which was relieved by the use of ordinary emollients such as ung. zinci. Case $\mathrm{F}$ seems to have induced a pustular dermatitis. These dermatites were doubtless due to the enthusiastic scrubbing and inunction. Case $\mathbf{A}$ is of special interest. After spending three days in bed in a state of acute discomfort (all the while treating himself with a sulphur ointment) he developed a diffuse dermatitis which affected all parts of the body, including the face. This was succeeded by a pompholyx due to a streptococcal infection from which he was still suffering more than three weeks after the incidence of the infection. In this case there is also a history of an attack of scabies during infancy.

Microscopy. The carcass was scraped on the Sunday morning-i.e., 24 hours after death. Sarcoptes scabiei were found to be present in abnormally large numbers.

One concludes from the above observations that the mites migrated from a killed and chilling equine host to human hosts, passing through the clothing with little delay and setting up irritation in a period rarying from 2 to 24 hours.

\section{FURTHER EXPERIENCES WITH THE SCHICK TEST. ${ }^{1}$}

By R. A. O'BRIEN, M.D. MELb.,

A. J. EAGLETON, M.D., M.R.C.P. LOND.,

C. C. OKELL, M.R.C.P.LOND., A. T. GLENNY, B.Sc., AND

E. M. BAXTER, M.Sc. SHEFF.

(From the Wellcome Physiological Research Laboratories.)

THE work included 1150 tests. One group of 160 medical students contained only 56 " negative reactors" to the Schick test, giving the low rate of immunity of 35 per cent. ; amongst 22 nurses in the same hospital, only 45 per cent. were immune, whereas in a hospital staff of doctors, nurses, and attendants, consisting of 182 adults, 78 per cent. were immune. Zingher in New York noted that the higher the social stratum, the lower the immunity rate; the apparent explanation is that the wealthy protect their children from infection and therefore from natural active immunisation, while the poor cannot do so, and their children must either develop immunity or succumb to the disease. In a group of 734 children in an institution where diphtheria had recently occurred, 534 were immune-i.e., 73 per cent. Amongst the whole 1150 persons only 7 "positive (and pseudo)" or " combined" reactions were met with. The immunity rates at various ages agreed with those previously published. Amongst the children in the school blocks in which diphtheria had occurred, the immunity rate was not on the average higher than in other non-infected blocks. Children from the various blocks mingled in play. It was generally found that brothers and sisters gave the same reaction, and that if the younger were negative the older were also negative. Amongst 87 families, in six families all the children gave a positive reaction; in 51 all were negative; while in 13 families the older children were negative and the younger positive. "Zingher's law" was contravened in 16 families, where the younger were negative and the older positive.

On this point our observations are not of great value, since brothers and sisters are divided amongst various school blocks at an early age.

The error involved in making a decision 24 hours after the test was about 5 per cent. Amongst 238 children, records were made of the first day's reading and of subsequent readings. In 10 the negative reading, made on the first day, became later positivei.e., these were delayed reactors. In 0.5 per cent. an initial "positive" proved later to be "negative." Four hundred and ten children were examined on the second day and again at a later date; three childreni.e., 1 per cent. - who were recorded as negative on the second day, later developed positive reactions. Thus if an epidemic occurs in a school, it appears to be practicable to apply the Schick test to all the inmates, and next morning to decide with a $9 \tilde{x}$ per cent. accuracy who are positive reactors and need passive immunisation or careful daily examination, until active immunisation can, if desired, be carried out.

The error of the test is very small ; if readings can be made on several different days, and in the rare doubtful cases the test, repeated, an accurate decision can be arrived at in practically 100 per cent. of cases ; if one is able to titrate the blood in the fractional percentage of cases that still cause some hesitation, the percentage of accuracy will reach 100 . In practice it is very easy to read accurately about 95-98 per cent. of patients, but the remaining few persons demand careful examination. Nearly always when one is in doubt the patient is really immune. The readings in 24 cases in the whole series of 1000 cases caused some difficulty. In 23 of these the final readings of the repeat Schick test as well as the blood titration showed that the patient was immune. Eleven samples contained between 1/10 and 1 unit per c.cm., 11 contained 1 Abstract of a paper read at a meeting of the Section of
Pathology of the Royal Society of Mredicine on April 4 th. 